\author{
சு. சந்திரகுமார் அ, *
}

அ நுண்கலைத்துறை, கிழக்குப் பல்கலைக்கழகம், வந்தாறுமூலை, இலங்கை

\title{
The Human Investment in Local Art Entrepreneurship: A Study Based on
}

\section{Palm Products}

\author{
S. Chandrakumar a, ${ }^{*}$ (i) \\ a Department of Fine Arts, Eastern University, Vantharumoolai, Sri Lanka.
}

Corresponding Author: srckumar20@yahoo.com

Received : 28-10-2020 Accepted : 04-12-2020

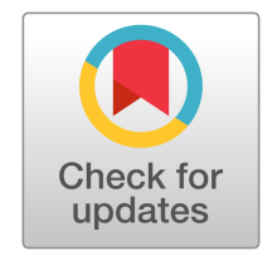

\begin{abstract}
:
There is an illusion in the neoliberal world that only when the production is implemented through mechanization there will be growth and progress. Because of this reason, people have become consumables, and the trend and uniqueness to think and act upon one's own has become rare. In addition to this, the production of machineries affects the environment and human life, and it costs a lot of money to get rid of these affects. Nevertheless, when the human resource and its effort are utilized, the aspects such as self-thinking, researching skills, sensible creative arts without having vague ideas are developed. Further, this leads to increase the connection with the producers and to enhance the environment friendly situation. It is important to know that these kinds of sustainable local employments exist in our socio-cultural space. The entrepreneurship through palm products is significant among them. These salable products are closely connected to the life of the people. This production is done using the skills of the people within the village according to the level of requirement and it is also done in the Palm products production centers through government Departments. This is being carried out in our area where the palm resources exist. It is vital to have self-confidence, diligence and aim in the local art entrepreneurship. In this activity, the situation where there was sharing, directly selling the products to the consumers have changed to a situation where the capitalists purchase the products. The initiation of human involvement is clearly exposed in the trend of entrepreneurship of palm products. This study is based on the expression of this art entrepreneurship in Batticaloa, Sri Lanka.
\end{abstract}

Keywords: Human resource, Art Entrepreneurship, Palm products, Mechanization, Consumption.

\section{ஆசிரியர் குறிப்பு}

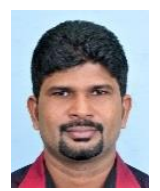

\section{திரு.சு.சந்திரகுமார், இலங்கை கிழக்குப் பல்கலைக்கழகத்தில்}

நுண்கலைத்துறையில் நாடகமும் அரங்கியலும் பாடத்தில் முதுநிலை விரிவுரையாளராகப் நாடகச் செயற்பாட்டாளரான இ இவர், பாரம்பரிய நவீன அரங்குகளில் 
காலனிய நீக்கம், பின்நவீனத்துவம், நிகழ்த்துகை மையம் சார்ந்த கோட்பாடுகளினூடாக நவீன அறிவின் அதிகாரம் அரங்கில் பிரதிபலிப்பதை கட்டவிழ்த்து ஆராய்ச்சிகளை முன்னெடுப்பவர். அரங்கைக் கற்பிக்கும் முறையில் ஆற்றுகை மையக் கற்றல் - கற்பித்தல்' எண்ணக்கருவை உருவாக்கி செயற்படுத்தி வருபவர். பாரம்பரிய அரங்கை அதன் வெளியில் பயில்வதற்கும், மேம்படுத்துவதற்கும் 'அடிப்புறத்தில் இருந்து அடிப்புறத்திற்கு' எனும் கோட்பாட்டை உருவாக்கிச் செயற்படுத்துபடும் பேராசிரியர்.

\section{முன்னுரை}

கலைத் தொழில்முனைவு மூலமான பொருட்கள் பல வழிகளில் சமூக, பொருளாதார பண்பாட்டுத் தளங்களில் முன்னெடுக்கப்படுகின்றன. மானிட வாழ்வில் இதற்கென தனித்துவமான இடம் உண்டு. இத் தொழில்முனைவு பொருளாதாரத்தில் மட்டுமன்றி, சமுதாய பண்பாட்டு நிகழ்வுகளிலும் அடிப்படையாக உள்ளது. இதனைச் சிறு கைத்தொழில், குடிசைக் கைத்தொழில், அலங்காரப் பொருட்கள் என்றும் அழைப்பர். மட்டக்களப்பில் பல மூலப் பொருட்களைக் கொண்டு கைவினைக் கலைப் பொருட்கள் உற்பத்தி செய்யப்படுகின்றன. இவற்றுள் பனை ஓலைப் பொருட்கள், பிரம்பு வேலைப்பாடுகள், களி மண்ணினால் தயாரிக்கப்படும் பொருட்கள், மரத்தாலான அலங்கார வேலைப்பாடுகள், தும்புப் பொருள், உருக்கு வேலைப்பாடுகள் என்பன அடங்கும். இதில் உற்பத்தி செய்யப்படுவதற்கும் அந்த மக்களின் பண்பாட்டு வாழ்வியல் ம முறைக்கும் உயிர்த்துடிப்பான ஊடாட்டம் நிலவுகின்றன. 'மூலதனமானது பொருளாதார பரிமாற்று செயல்முறைக்குள்ளேயே உள்ளது. இது சுழற்சி முறையில் வளரும் செல்வம்' (Karl Marx, 1998) என மார்க்ஸ் குறிப்பிடுவது போல கைத்தொழில் உற்பத்திகள்மூலம் கிடைக்கும் பணத்தைக் கொண்டு மீண்டும் மூலப் பொருட்களைப் பெற்று கலைத் தொழில்முனைவுக்கான உற்பத்திப் பொருட்களத் தயாரிக்கின்றமை குறிப்பிடத்தக்கது.

நவீன கல்வியின் பார்வையில் இவை பொருத்தமற்ற பொருளாதாரம், விரைவாக இலாபம் கிடைக்காது எனும் கட்டமைப்பு விதிக்கப்பட்டு அதுவே இளம் தலைமுறையினருக்கு முன்னிலைப்பட்ட பதிவாகவும் நிலவுகின்றது. மனித மூல வளத்தை அடிப்படையாகக் கொண்ட உள்ளூர் அறிவு, திறன், அழகியல் அம்சங்களைத் தன்னகத்தே கொண்டு உற்பத்தி செய்யப்படும் பனை ஓலைக் கலைப் பொருட்கள் சுய ஆக்க வெளிப்பாடாகவும், மகிழ்ச்சியைத் தருவதாகவும், கற்பனை வளத்தை விரிவுபடுத்தித் தங்கி வாழும் நிலமையை ஒழிப்பதாகவும், பண்பாடுசார்ந்த கற்றல் செயல்மையமாகவும் பயில்நிலையில் இருந்து வருகின்றது. ஆனால், காலனிய நவீன அறிவுப் பரவலாக்கம் இயந்திர உற்பத்தி முறையைப் பிரதானப்படுத்தியதால் மனித மூலவளம் குறைக்கப்பட்டு, பனம்பொருள்சார் கலைத் தொழில்முனைவு பாதிக்கப்பட்டாலும் சமகாலம் வரையும் உற்பத்திச் செயற்பாடுகள் முன்னெடுக்கப்பட்டு வருகின்றன. இதனை வலுப்படுத்துவதன் மூலமே நிலைத்து நிற்பதற்கான மேம்பாட்டினை அடையமுடியும்.

மட்டக்களப்பில் பனம்பொருள்சார் கலைத் தொழில்முனைவில் மனித மூலதனச் செயற்பாடுகள் மட்டக்களப்பில் உற்பத்தி செய்யப்படும் கலைத் தொழில் பொருட்களில் 
படைப்பாளர்களின் திறன், உணர்வு, அழகியல் நட்பங்கள், வருமானம், கற்பனை படைப்பாக்கம் போன்றவை முக்கியமானவையாக உள்ளது. இவை மாத்திரமன்றி, மனித மூலதனத்தினை அடிப்படையாகக் கொண்ட பண்பாட்டுத் தளத்தில் அதற்கான வேலைப்பாடுகளும் பாரம்பரியமாக நடை முறையில் இருந்து வருகின்றன. இப்பிரதேசத்தில் பரம்பரை பரம்பரையாக மக்களின் வாழ்வியலோடு முன்னெடுக்கப்படும் தொழிலாக இது செயற்பட்டமை அறிதலுக்குரியது.

\section{பனை வளம்}

இலங்கையில் வடக்குக் கிழக்கு மாகாணங்களிலேயே மிகவும் அதிகமா

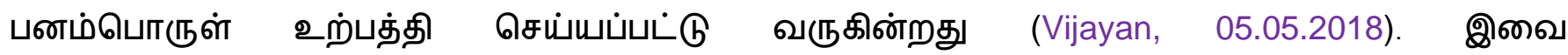
வருமானத்தைத் தரும் ஒன்றாக இருப்பதோடு, மனித மூலவளத்தின் உழைப்பிற்கும், அழகியல் வெளிப்பாட்டிற்கும், மனித படைப்பாக்கம்சார் திறன் வெளிப்பாட்டிற்கும் சூழல்நேய தொழில் முற்சியாக அமைகின்றது. 'மட்டக்களப்பில் யானைகள் வரும் எல்லைக் கிராமங்களில் நெருக்கமாகப் பனை நாட்டப்பட்டு பராமரித்தால் யாானைகள் வந்து கிராம வாசிகளைத் தாக்குவது தடைப்படும் $\quad$ (Thangaraja, 14.06.2018) என விவசாயிகள் கூறுகின்றனர். இது மண்ணரிப்பைத் தடை செய்து சூழலையும் சம நிலையாக்க உதவுகின்றது. பனை மரங்கள் நாளுக்கு நாள் வளர்ச்சி என்ற பெயரில் வெட்டி அழிக்கப்படுகின்றமை மிகவும் துன்பகரமான செயற்பாடாக உள்ளது. இதனால், 'மட்டக்களப்பு பனை அபிவிருத்திச் சங்கம் ஒரு இலட்சத்திற்கு மேலான பனங் கன்றுகளை நாட்டியுள்ளது' (Vijayan, 05.05.2018) என குறிப்பிடப்படுகிறது.

\section{பனை ஓலைசார் கலைத் தொழில்முனைவில் மனித மூலதனம்}

மட்டக்களப்பில் பனை ஓலையினை அடிப்படையாகக் கொண்ட கலைத்

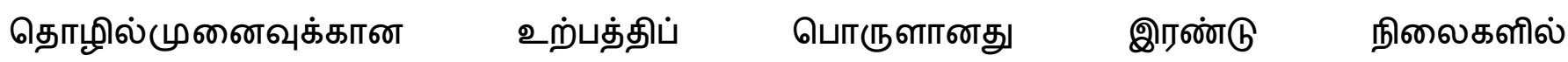
முன்னெடுக்கப்படுகின்றன.

1. பரம்பரை பரம்பரையாக மக்கள் சார்ந்து அவர்களே தமது தேவைக்காக உற்பத்தியில் ஈடுபடுவது.

2. பயிற்சி நிலையங்கள் ஊடாக அலங்கார நுட்பம், பொருளாதார வலுப்படுத்தல் என்பவற்றிற்கு முன்னெடுப்பது.

இந்த முன்னெடுப்புக்கள் இரண்டும் இங்கு வாழும் ஊர் மக்களால் பரவலாக நடைமுறையில் உள்ளன. மூதாதையர் கலைப் படைப்பாக்க அறிவு, திறன் மூலம் ஆழமாகவும், அழகாகவும் திட்டமிட்டு தமது கற்பனையை விரிவுபடுத்தி பல பொருட்களைப் பனம்பொருட்களால் படைத்துள்ளனர். அப்பொருட்கள் வாழ்வியலிலும், உழைப்பிலும் முன்னிலை வகித்தது. பருவகால நிலமானியத் தொழிலைச் செய்து தமக்குக் கிடைக்கும் ஓய்வு நேரங்களிலும், செய்யும் தொழிலின் தேவைக்காகவும் இதனைத் தயாரித்தனர். இவற்றுள் பெட்டி, கைப்பெட்டி, சுளகு, பாய் போன்றவை முக்கியமானவை. சேனைப்

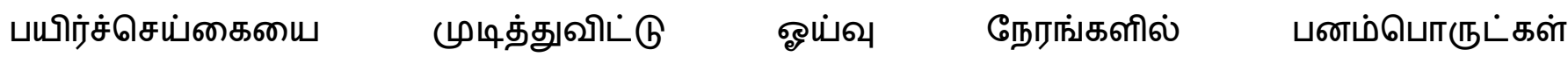


தயாரிக்கப்பட்டுள்ளமை குறிப்பிடத்தக்கன. தமது உற்பத்திப் பொருட்களைச் சேகரிப்பதற்காகச் சுயமாகத் தாமே உற்பத்தி செய்தமை, உள்ளூர் கலைத் தொழில்முனைவின் ஊடான வாழ்வியல் மகிழ்வை வெளிப்படுத்துகின்றது. நிலமானிய சமூக அமைப்பில் இப்போக்கு இன்றும் பயில்நிலையில் உள்ளது. ஏனெனில், பனம் பொருட்களுக்கும் பண்பாட்டிற்கும் இடையில் நெருக்கமான இடையூடாட்டம் உண்டு. பல்தேசியக் கம்பனிகளின் இயந்திர உற்பத்திமூலம் ூூழலுக்குப் பொருத்தமில்லாத இரப்பர் பொருட்கள் பரவாலக்கப்படுவதும் நிலைகொண்டுள்ளது.

சமகாலத்தில்

தொழில்முனைவுக்கான கலைப் பொருட்கள் மனித மூல வளத்தை அடிப்படையாகக் கொண்டு உற்பத்தி செய்யப்படும் போக்கும் உருவாகியுள்ளது. இவை வருமானத்தை மேம்படுத்த முன்னெடுக்கப்படுகின்றன. இவை நவீன கல்வி முறைக்குள் மிகவும் திட்டமிட்டு மேற்கொள்ளப்படுகின்றன. தற்காலத்தில், இந் நிலையங்களை மையப்படுத்தி

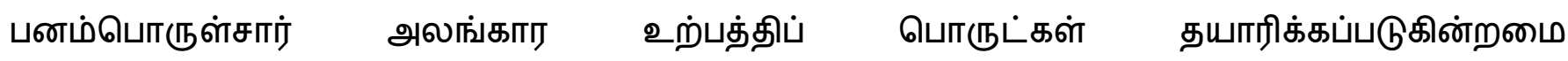
அறிதலுக்குரியது. இதில் கலை ஆற்றலை மனித மூலதனத்துடன் வெளிப்படுத்தினாலும் இடைத்தரகர்களை உருவாக்கிச் சிறு முதலாளித்துவத்தை மட்டும் வலுப்படுத்த வழிவகுக்கின்றது. இதனால் பண்பாட்டிற்கும் இவ் உற்பத்திக்கும் அந்நியப்படுத்தல் வருவதோடு, வருமானமும் பாதிக்கப்படுகின்றது. இது மனித மூலதன பேண்தகு பொருளாதாரத்தைக் குன்றச் செய்கின்றது.

\section{மனித மூலதனத்தை வலுப்படுலுத்துவதில் பனம்பொருள் உற்பத்தி நிலையம்}

மட்டக்களப்பு நகருக்கு கிழக்கே சுமார் 13 கி. மீ தொலைவில் அமைந்துள்ள பாலமீன்மடு எனும் இடத்திலே பனம்பொருள் உற்பத்தி நிலையம் அமைந்துள்ளது. இங்கு பனம்பொருள் உற்பத்தியினுடனான கலைத் தொழில்முனைவுக்கான பல முன்னெடுப்புக்கள் நடைபெறுகின்றன.

இத்தலைமைக் காரியாலயத்தின்கீழ் பயிற்சி நிலையங்கள், உற்பத்தி நிலையங்கள், விற்பனை நிலையங்கள் ஆகியவை பெண்களை மையப்படுத்தி செயற்படுத்தப்படுகின்றன. இங்கு பனம் பொருட்களைக் கொண்டு தயாரிக்கும் பத்துப் பயிற்சி நிலையங்கள் உள்ளன. அந்தப் பயிற்சி நிலையங்கள் ஓந்தாச்சிமடம், மாங்காடு, தாளங்குடா, நாவற்குடா, ஆறுமுகத்தான்குடியிருப்பு, மயிலம்பாவெளி, முறக்கொட்டாஞ்சேனை, சித்தாண்டி வாலக்காலை, நாசிவன்தீவு ஆகிய இடங்களில் இயங்குகின்றன. இவ்விடங்கள் மூலப் பொருட்கள் கிடைக்கும் பகுதிகளாக அமைகின்றன. ஆனால், இப்பயிற்சி நிலையங்கள் படுவான்கரையிலும் ${ }^{1}$, எழுவான்கரையில் வாகரை போன்ற மிகவும் போரினால் பாதிக்கப்பட்ட பிரதேசங்களிலும் குறைவு. எனினும் இங்கு இரண்டு விற்பனை நிலையங்கள் உண்டு.

\footnotetext{
1 மட்டக்களப்பு வாவியின் கிழக்குப்பகுதியில் சூரியன் உதயமாவதனால் எழுவான்கரை என்றும், மேற்குப்பகுதியில் சூரியன் மறைவதனால் படுவான்கரை என்றும் அழைக்கும் மரபு உண்டு. இவை காரணப்பெயர்களாகும்.
} 
கிராமியப் பொருளாதார மேம்பாட்டு அமைச்சு தனது 2016 ஆம் ஆண்டிற்கான

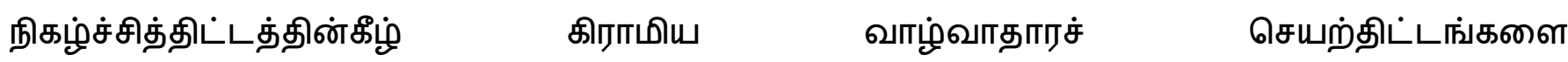
நடைமுறைப்படுத்துகின்றது. இதன்கீழ் 2016 ஆவணி தொடக்கம் மார்கழி வரை ரூபாய் 300 மில்லியன் ஒதுக்கீடுகள் அநுராதபுரம், பொலனறுவை, மட்டக்களப்பு ஆகிய மாவட்டங்களுக்கு பகிர்ந்தளிக்கப்பட்டு, குறிப்பிட்ட மாவட்டங்களில் நிலவும் வள முரண்பாடுகளைத் தீர்க்கவும், கிராமக் கைத்தொழில் முன்னேற்றத்தை மேம்படுத்தவும் வழிசமைக்கப்பட்டுள்ளன.

இவ்வாறான நிகழ்ச்சித்திட்டங்கள் மட்டக்களப்பு மாவட்டத்தின் பனம்பொருள்சார் கைத்தொழிலில் ஈடுபாடுகாட்டும் மனித மூலதனங்களுக்கும் சிறப்பான எதிர்காலத்தினை

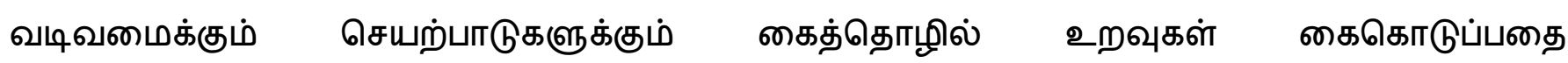
எடுத்துக்காட்டுகின்றன.

\section{மனித மூலதன வலுவாக்கத்திற்குப் பயிற்சி வழங்கப்படும் முறை}

பயிற்சி நிலையங்களில் ஆறு மாதம் பொருத்தமான ஆசிரியர்களுடாகப் பயிற்சி வழங்கப்படும். பயிற்சி பெற்றவர்கள் பின்னர் தயாரிப்பாளர்கள் அல்லது உற்பத்தியாளர்களாக மாறுவர். பயிற்சிக் காலத்தில் மூன்று மாதங்கள் கடந்ததுமே அவர்கள் நிலையத்தினூடாக கலைத் தொழில்முனைவுக்கான உற்பத்தியினை ஆரம்பிப்பர். கலை அழகியலுக்கான நுட்பமுறைகள், அளவுத் திட்டங்கள் என்பன செயற்பாட்டினூடாக வரன்முறையாக கற்பிக்கப்படும். கலைத் தொழில்முனைவுக்கான பயிற்சி பெறுனராக பெரும்பாலும் கிராமத்தில் இருக்கும் பெண்களே உள்ளீர்க்கப்படுகின்றனர். இதில் வீட்டில் ஏ ஏற்கனவே இந்த அலங்கார வேலைகளில் ஆர்வமுடையவர்கள், புதிதாகப் பழக விரும்புபவர்கள், வேலையில்லாது வீட்டில் இருப்பவர்கள் எனப் பலர் ஈடுபடுகின்றனர். ஆனால், வறுமைக் கோட்டின்கீழ் இருந்து பயிற்சி பெற்ற பெண்கள் பயிற்சியின் பின்னரும் முன்னைய திறமையையும் கற்பனை வளத்தையும் பயன்படுத்தி, தொடர்ந்தும் உள்ளுர்த் தொழில்முனைவில் ஈடுபட்டுக் கைவினைக் கலைப் பொருட்களை உற்பத்தி செய்கின்றனர். செய்து செயற்பட்டுக் கற்றல், தமது கிராமத்தவருடன் சேர்ந்து கற்றல் போன்றவை மூலதனத்திற்கான பேண்தகு உற்பத்தி முறைமையை அதிகரிக்கும். அடிப்புறத்தில் இருந்து அடிப்புறத்திற்கு (Bottom to Bottom) வலுப்படுத்தப்படும்போதே உள்ளூர் மனிதவளம் மேம்படுத்தப்பட்டு விரிவாக்கம் பெறும்.

மட்டக்களப்பில் இரண்டு வீதமான பனம்பொருள் பாவனை உள்ளது. இது பத்து வீதமாக அதிகரிக்குமாயின் ஒரு கோடி ரூபா வருமானம் வருடத்திற்குக் கிடைக்கும் (Vijayan, 05.05.2018). இதை தென்னிலங்கை மக்களும், வெளி நாட்டவரும் தமது பண்பாட்டுப் புழக்கத்திற்காக அதிகம் வாங்குகின்றனர்.

\section{பனம்பொருள்சார் தொழில்முனைவின் மனித மூலதனக் கையாள்கை} முறை

கைவினைக் கலை மனித உழைப்பின் கற்பனை, அழகியல் ஆகியவற்றின் அடியாகவே முன்னிலைப்படுத்தப்படுகின்றது. உற்பத்தி செய்யும் கைவினைப் பொருள் 
அதனூடாக பெறப்படும் வருமானத்தை மையமாகக் கொண்டு ம முன்னெடுப்பதினூடாகவே கலை வெளிப்பாடு பிரதானப்படுத்தப்படும். இங்கு மனித மூலதனத்தின் அழகியல் உணர்வு (Aesthetic Sense) வெளிப்பாடு கலை உணர்வினூடக மேலேழுவது கவனத்திற்குரியது. இதற்குச் சமூகத் தேவையுண்டு. குறிப்பாக, இவை சடங்குகள், விழாக்கள் முதலான சமயம்சார்ந்த நிகழ்வுகளில் தங்கியுள்ளன.

அத்துடன், உற்பத்தி நிறுவனங்களினால் பிறப்பிக்கப்படும் கொள்வனவுக் கட்டளை (order) கிடைக்கும்போதும் கைவினையின் தரம் நிர்ணயிக்கப்படும். கிராமங்களி கலைத்தொழில் முனைவின் அடிப்படையில் உற்பத்திக்காலம் தீர்மானிக்கப்படும். தேவைக்கேற்ப இரவு, பகலாகத் தம்மை ஈடுபடுத்தி பொருட்கள் உற்பத்தி செய்யப்படுகின்றமை குறிப்பிடத்தக்கன (Ranji, 12.05.2018). பனம்பொருள்சார் னைவினைப் பொருட்களின் அலங்கார வேலைப்பாடுகளில் பணம் முதலீடாக அமைந்தாலும் அதனுள் மனித அழகியல் உணர்வு (Human Aesthetic Sense) நிறைத்துள்ளமை மறுக்க முடியாதது. இவ் அழகியலே மனித நுகர்வை அதிகரிக்க வழிவகுக்கின்றது.

பனை ஓலைகளை வெட்டித் தயார் படுத்துவது பற்றி உள்ளரர் உற்பத்தியாளர் குறிப்பிடுவது கவனத்தில் கொள்ளத்தக்கது. பனை ஓலையை வெட்டி அதனைக் காய விட்டுத் தேவையான அளவிற்கேற்ப வார்ந்தெடுத்து பல வர்ணங்களைக் கொண்ட சாயமிட்டுக்கொள்வது ஆரம்பகட்ட வேலைப்பாடுகளாகும் (Parimalam, 12.05.2018). குறிப்பாக, இவற்றில் பதமாகக் காயவைத்தலும் அதனைத் தாமே கவனமாக எடுத்து பாதுகாத்தலும் முன்னிலை வகிக்கின்றது. ஒவ்வொரு ஓலையையும் கிழித்தெடுத்தல், பின்னர் அளவிற்கேற்ப வார்ந்தெடுத்தல் ம்க்கிய செயற்பாடாகும். ஓலையில் இருக்கும் நூல் மாதிரியைத் தனியாகப் பிரித்து பூக்கள் தயாரிப்பதற்கு எடுக்கப்படுகின்றது.

தங்களால் தயாரிக்கப்படும் பொருட்களை அடிப்படையாகக் கொண்டு 'வர்ணக் கலவை' செய்யப்படுகின்றது. இயற்கையாகச் சாயங்களைப் பயன்படுத்தி தமது படைப்பாக்கத்தைச் சூழல் நட்புடன் முன்னெடுக்கின்றமையும் குறிப்பிடத்தக்கது. அத்தோடு, ஆரம்பத்தில் கோழிச் சாயம் பயன்படுத்தப்பட்டது. இதில் பச்சை, செம்மஞ்சள், மஞ்சள், சிவப்பு போன்ற வர்ணங்களே அதிகம் உபயோகிக்கப்பட்டுள்ளது. இது கலை நுணுக்கத்துடன் தமது கற்பனையூடாக வெளிப்படும்.

பூக்களுக்குரிய இதழ்கள் எனின் அவை நேர்த்தியாக வெட்டி எடுக்கப்படு, பின் அந்த இதழ்கள் தேையான அளவு வர்ணச் சுடுநீரினுள் வைத்து நனைக்கப்படும். அவை உடனே நற்தண்ணீரில் கழுவப்படும். அப்போதுதான் வர்ணம் அவ்வோலையில் பற்றியிருக்கும். அவற்றைக் கடும்வெயிலில் காயவிடாது காற்றுப்படக்கூடிய இடத்தில் உலரவைத்துக் கொள்வர் (Ranji, 12.05.2018). இதற்கு மனித மூலதனமே பிரதானமாக அமைகின்றது. இதில் சமுதாய ஆளுமையே அதிகம் பிரயோகப்படும். 'எந்தவொரு உற்பத்தி செய்த பொருளைக் கொண்டும் அது பொருளாதார ரீதியாக பயனுள்ள வகையில் செயற்பட்டால் அதன்மூலம் நபரின் உற்பத்தித் திறனை அதிகரிக்க முடியும்' (Karl Marx, 1998) எனும் கார்ல் மார்க்ஸ் இன் கருத்து இச்செயற்பாடுகளுடன் பொருத்திப் பார்க்கக் கூடியதாக உள்ளது. 
பெற்றிக் வர்ணம் கொண்டு அலங்காரப் பொருட்களைத் தயாரிக்கும் நுணுக்கமும் உண்டு. கூடை (Basket) தயாரிக்கும்போது இந்த அழகியல் வெளிப்பாடு பயன்படும். ஒரு ஓலையில் பல வர்ணங்கள் சேர்க்கப்படுவது பெற்றிக் ஆகும். தேவையான அளவு வார்த்தெடுத்த ஓலையைக் கொதித்துக் கொண்டிருக்கும் நீரில் ஒரு வர்ணத்தை இட்டு குறிப்பாகப் பச்சை வர்ணத்தை இட்டு கலக்கி ஓலையின் தேவையான பகுதி மட்டும் நனைத்தெடுக்கப்படும். பின்னர் மஞ்சள் வர்ணத்தைப் போட்டுக் கலக்கி அந்த ஓலையின் அடுத்த பக்கம் நனைத்தெடுக்கப்படும். நீல வர்ணம் தேவையாயின் அதன் மறு பக்கம் நனைக்கப்படும். இதனால், பொருத்தமான நிறத்தைக் கொண்டு 'பெற்றிக் வர்ண வடிவம்' எதிர்பாராத அழகுடன் காட்சிதரும். இதனைக் கொண்டு பொருட்கள் தயாரிக்கப்படுகின்றன. இவை வர்ணங்களின் பயன்பாடு ஆகும் (ஆய்வாளரின் நேரடி அவதானம்).

\section{பனம்பொருள் உற்பத்திப் பொருட்களின் அழகியலும் அதன் அளவுத் திட்டமும்}
அலங்காரப்
பொருட்களை
வடிவமைப்போர்
பொருத்தமான
அளவுப்

பெறுமானங்களுடன் தயாரிப்பர். கூடை, பெட்டி, தொப்பி, பூச்சாடி, பூக்கள், மாலை முதலான பொருட்களுக்கென முறையான அளவுமுறை உண்டு. அங்குலத்திலே இ இ அமைந்திருக்கும். கலைத் தொழில்முனைவு உற்பத்தியாளர்கள் இவற்றை மிக நுணுக்கமாகவும், அவதானத்துடனும் பயன்படுத்துவர். சரியான அளவுடன் தரமாகத் தயாரிக்கும் பொழுதே அதன் முடிவுப் பொருட்கள் 'உற்பத்தி நிலையத்தில்' இருந்து கொள்வனவு செய்யப்படுகின்றது.

பின்னல் வேலையுடைய கைவினைப் பொருள் தயாரிக்கப்பட வேண்டுமாயின் அக்கலைஞரால் மிக நுணுக்கமாகப் படிப்படியாகத் தயாரிக்கப்படும். இதில் ஈடுபடுவோர் பொருத்தமான வர்ணங்களைச் சேர்த்து அழகுபடுத்துவர். அதனை ஞாபகத்தில் வைத்திருந்து பின்னலை வர்ணங்களுடன் சேர்த்து அலங்கரித்தல் முக்கிய திறனாகும். பின்னல்களில் சாதாரண பின்னல், வாழை முடிச்சிப் பின்னல், கன்னி முடிச்சுப் பின்னல் எனப் பலவுண்டு. இதனைக் கருத்திற்கொண்டு பின்னல் வேலைப்பாடுகள் இடம்பெறும். தொழில் முனைவோர் அவற்றைக் கொண்டு கூடை, பெட்டி என்பவற்றைத் தயாரிப்பர். ஈக்கில் வைத்தே பின்னல் வேலைப்பாடுகள் செய்யப்படும். பூமாலை செய்யும்போது அதற்குரிய இதழ்களைச் சரியாக வெட்டிக்கொள்வது அவதானமாக இடம்பெறும். பெரியதொரு பூங்கொத்து ஒன்றினை அலங்காரமாகக்கொண்டு வருவதற்கு இதழ்களை வெட்டி வளைத்தல் அல்லது சுருண்டதாக்குதல் அழகைத்தரும். இது இளஞ்சூட்டுக் கம்பித்துண்டினால் இஸ்த்திரிசெய்து (Iron) வளைக்கப்படும். இவை சூட்டினால் வளைந்ததும் அவற்றை எடுத்து வர்ணங்களுக்கேற்ப கோர்த்து அழகான பூமாதிரியான கொத்தைத் தயாரிப்பர் (ஆய்வாளரின் நேரடி அவதானம்).

இங்கு பொருட்களின் அளவு முறை, வர்ணப் பயன்பாடு, அதன் முடிவு (Finishing) வார்ந்தெடுத்தல், சரியாக இஸ்த்திரியிடல் (Ironing) போன்றவை மனித மூலதனத்தின் முக்கிய வெளிப்பாடாகும். 
பாயினை இழைக்கும்போதும், பெட்டிகளைப் பின்னும்போதும் வர்ணஓலைகளின் இணைவுடனான வடிவமைப்பு பனை ஓலைக் கைவினைப் பொருட்களில் வரும் இடம் முக்கியமாகும். அவை பொருத்தமாக இணையாவிட்டால் விற்பனையாகாது. இதனால் கற்பனை வளத்துடன் இதனை இணைத்துப் பார்ப்பர். சாதாரணமாகப் பனை ஓலைப் பெட்டியை இ இ இகைகும்போது அவற்றில்வரும் வர்ணக்கோடுகளின் இணைவு வார்ந்தெடுக்கப்பட்ட வர்ண ஓலைகளின் சேர்க்கையில் தங்கியுள்ளது. இந்த உத்திமுறை குறிப்பிடத்தக்க கலை உணர்வு வெளிப்பாடாகும். இவ் மனித அறிவின் நுட்பம் தொழில்முனைவில் நகர்வோரைக் கவர்வதற்கும் ஏனைய பொருட்களுடன் போட்டி போடுவதற்கும் வழிவகுக்கும் என்பதைச் சுட்டுகின்றது.

கலைத் தொழில்முனைவின் உற்பத்தித் தளத்தில் பனை ஓலைமூலம் பொருட்கள் தயாரிக்கப்படும்போது அதன் மனித மூலதனமாகப் பெண்களே அதிகமகத் தொழிற்படுகின்றனர். பொருட்கள் மிகவும் நுணுக்கமாகவும் அலங்காரமாகவும் உற்பத்தி செய்யப்படுகின்றன. இவற்றுள் வருமானம், முதலீடு என்பன பிரதானமாக இருந்தாலும் அலங்காரமான கைவினைக் கலையாகவே உள்ளது. மான், பறவைகள் போன்ற பொருட்களைத் தயாரிப்பது அவர்களின் கற்பனை வளத்தின் ஆழத்தைப் பிரக்ஞ பூர்வமாக வெளிக்கொணர்கின்றது.

கலைத் தொழில்முனைவில் தயாரிக்கப்படும் கைவினைப் பொருட்களில் பெட்டி வகைகள், பூக்கள், கூடை வகைகள், மெத்தம் (பூவைத்து கொழுவுவது), கோட்டுன், மேசைத் தட்டு (ஏழு வகைத் தட்டு உண்டு), பூச்செண்டு, மாலைகள், கைப்பைகள், தொப்பி வகைகள், பாய் வகைகள் போன்றன பிரதானமானவையாகும்.

இந்த உற்பத்தி முறையுடன் பயிற்சி பெற்றவர்கள் மிகவும் ஆர்வத்துடன் தம்மை ஈடுபடுத்தி முன்னெடுக்கின்றனர். தமது வீட்டு வேலைகளுடன் ஓய்வு நேரங்களிலோ அல்லது கேள்வி நிலவும்போதோ அல்லது பண்பாட்டு விழாக்களிலோ தொடர்ச்சியாக தமது தொழிலின் நிமிர்த்தம் பொருட்களைத் தயாரித்து விற்பனை செய்கின்றனர். கலைத் தொழில்முனைவின் சந்தைப்படுத்தலில் பொருட்களைக் கோருபவர்கள் வீட்டிற்கு வந்து சேகரிப்பதும் வழக்கமாக உள்ளது.

\section{மனித மூலதனத்தின் படைப்பாக்க உருவாக்கத்தில் அலங்காரப்}

\section{பொருட்கள்}

பனம்பொருட்கள் மூலமான கலை அழகுடன்கூடிய தொழில் முன்னெடுப்புக்களில் ஆற்றலையும் திறனையும் வெளிப்படுத்திப் பொருத்தமான வடிவங்கள், நிறம், அளவுத் திட்டம் ஆகியவற்றுடன் தயாரிக்கப்படும் அலங்காரப் பொருட்கள் சமுதாய ரசனையை அதிகரிக்கின்றது. வீட்டுச் சாளரத்தின் (Curtain) தொங்கவிட்டும், பூச்சாடியில் பூங்கொத்தாகவும், அன்பளிப்பாக பூங் கொத்தைக் கொடுத்தும் மகிழ்வதற்குரிய அலங்காரப் பொருட்களும் தயாரிக்கப்படுகின்றன. அவ்வாறே, சந்தையில் பொருட்களைக் கொள்வனவு செய்வதற்கான கூடை, பூப் பறிப்பதற்கான கூடை, காரியாலயத்திற்குக் கொண்டு செல்வதற்கான கைப்பை (Hand Bake), கோப்பு (File), உணவு எடுத்துச் செல்வதற்கான கைப்பை போன்றவையும் மிக 
நுணுக்கமாகப் பனை ஓலைமூலம் தயாரிக்கப்படுகின்றது. உணவுப் பொருட்களை வைத்து மூடுவதற்கான நீண்ட பெரிய கூடையும் பயன்படுத்தப்படுகின்றது. பாத்திரங்களை மூடுவதற்கான தட்டுக்கள், தேனீர்க் குவளைகளை உணவு மேசையில் வைப்பதற்கான தட்டுகள் போன்றவையும் பெருமளவில் தயாரிக்கப்படுவதைக் காணலாம். இதில் பழங்களை வைப்பதற்கான தட்டுகள், மூுபெட்டிகள் ஆகியனவும் குறிப்பிடத்தக்கன. கலையழகுடன்கூடிய திறனும், நுட்பமும் தொழில்முனைவு நிலையங்களில் முன்னிலை வகிக்கின்றமை குறிப்பிடத்தக்கது. இது மனிதத் திறனின் (Human Skill) வெளிப்பாட்டை அதிகரிக்கின்றது.

பனை ஓலைகளால் ரோஜா, அந்தூரியம், அலரி ஆகிய பூக்கள் அழகாகத் தயாரிக்கப்பட்டு, பச்சை, செம்மஞ்சள், ஊதா, சிவப்பு போன்ற வர்ணங்களால் அழகுபடுத்தப்படுகின்றன. அத்துடன், விளையாட்டுப் பொருட்கள், தொப்பிகள், கேக் கூடைகள் என்பன உத்தரவின்படி நேர்த்தியாகச் செய்துகொடுக்கப்படும். அத்துடன், பிட்டுக்குழல், அதனை இறக்கி எடுப்பதற்கான 'நீத்துப் பெட்டி' போன்றனவும் குறிப்பிடத்தக்கன. இங்கு பருவகாலத்திற்கு ஏற்ப உத்தரவு பிறப்பிக்கப்படும். இதனால், கலைத் தொழில்நுட்பமும் மனித படைப்பாக்கத்திறனும் ஆழமாக நுகர்வோரைக் கவரக்கூடியவாறு பயன்படுத்தப்படுகிறது.

இப்பனை ஓலைக் கைவினைப் பொருட்கள் சமூகத்தில் பண்பாட்டிலும், வாழ்வியலிலும், அலங்காரத்திற்கும், இதர தேவைகளுக்கும், அணிகலன்களுக்கும் பிரயோகமாகின்றன. எனவே, இப்பொருட்கள் சடங்கு, உணவு, பண்பாடு ஆகியவைபோல், அலங்காரப் பொருட்களாகவும் மனித மூலதனத்தை அடிப்படையாகக் கொண்டு படைக்கப்படுகின்றன. இதனூடாக, கேள்வி அதிகரிப்பதனால் கலைத் தொழில்முனைவின் பிரயோகம் விரிவாக்கம் பெறுகின்றது. இது கலைத் தொழிலமுனைவின் பண்பாட்டு உறவையும், பயன்பாட்டுத் தேவையையும், அதன் மனித மூலதனத்தின் இடையீட்டு விருத்தியையும் காட்டுகின்றது.

\section{கலைத் தொழில்முனைவில் பண்பாடும் பனம்பொருளும்}

கலைத் தொழில்முனைவில் வெளிப்படும் மனித மூலதனச் செயற்பாடு பண்பாட்டில் சமூக அழகியல் வெளிப்பாடாக உள்ளதனால் தொடர்ந்து பயில்வில் உள்ளது. சமுதாய ஆளுமை வெளிப்பாடே சமுதாய அழகியலைப் பண்பாட்டு மையம் நின்று கலைத் தொழில்முனைவைச் செய்வதற்கு உந்துசக்தியாக அமைகின்றது. பனை மரத்தி தோற்றுவாய் எப்போது இருந்ததோ அன்றிலிருந்தே சமூக பண்பாட்டு நிகழ்வுகளில் முக்கிய பொருளாக பனை ஓலை மூலமான கைவினைப் பொருட்கள் பயன்படுத்தப்பட்டு வருகின்றன. இதனால், சமூக பொருளாதாரமாக (Socio Economic) வலுப்பெறுகின்றது. இவை பண்பாட்டுப் பெறுமானம் மிக்கதாக உள்ளதால் இன்று வரையும் பயில்நிலையில் இருக்கின்றன. பண்பாட்டு வெளியில் (Cultural Space) இதற்கான படைப்பாக்க உணர்வு (Creative Sense) அதிகம் உண்டு. 
கைவினைக் கலைப் பொருட்கள் தமிழர் வாழ்வியலின் பண்பாட்டு வெளிப்பாட்டில் முதன்மை வகிக்கின்றன. இவற்றுள் களி மண்ணினால் தயாரிக்கப்படும் பொருட்கள், உலோக உருக்கு வேலைப்பாடுகள், நாணல், பனை ஓலை சார்ந்த தயாரிப்புக்கள், பிரம்பு வேலைப்பாடுகள், மரத்தாலான வடிவமைப்புக்கள் போன்றவை முன்னிலை வகிக்கின்றன. புதிய தொழில்நுட்பத்தின் வருகையுடன் வடிவமைக்கப்பட்ட இரும்பு, இரப்பர் பொருட்களின் தாக்கம் பண்பாட்டுத் தளத்தில் இடையூறு விளைவிக்கின்றது. எனினும், தமிழர் பண்பாட்டில் மக்கள் சார்ந்த கைவினைப் பொருட்களின் ஆக்கமும் பயன்பாடும் அதிகம் உள்ளது. ஏனெனில், இயந்திர உற்பத்தியில் இல்லாத மனித மூலதனத்தில் உணர்வு வெளிப்பாடு பின்னிப் பிணைந்திருப்பதனாலாகும். பண்பாட்டு வாழ்வியலில் இதற்கான தேவை இருக்கின்றது. இதனால், பனம்பொருளைக் கொண்டு உற்பத்தி செய்யப்படும் பொருட்கள் பண்பாட்டுப் பொருளாதார (Cultural Economic) உற்பத்தியாக அமைகின்றது. பண்பாட்டுப் பயன்பாட்டிற்காக உற்பத்தி செய்யப்படும் பொருட்களை அதன் உணர்வுடன் விற்பனை செய்வதும் அதனால் கிடைத்த பணத்தைக்கொண்டு உற்பத்தியில் மீண்டும் ஈடுபடுவதும் உள்ளூர் சமூகப் பொருளாதாரத்தில் கவனம் செலுத்த வைக்கின்றது. இதனால், மூதாதையர் பண்பாட்டு வாழ்வியலுக்குத் தேவையான பொருட்களைப் பனம்பொருளை முன்னிலைப்படுத்தி தாயரித்துள்ளனர். இவற்றுள் பாய், பெட்டி, கைப்பெட்டி போன்றவை முன்னிலை வகித்த பொருட்களாகும்.

மட்டக்களப்பில் மங்கல, அமங்கல, சமயம்சார் சடங்குகள், விழாக்கள், விரதம், திருமணம், சித்திரை வருடப் பிறப்பு போன்ற நிகழ்வுகளில் பனம்பொருட்கள் பயன்படுத்தப்படுகின்றது. உலகமயமாக்கல் சூழலில் இரப்பர் பொருட்களின் பாவனை பல்தேசியக் கம்பனிகளின் முகவர்களினூடாக முன்னிலை வகித்தாலும், உள்ளுர்க் கலைத் தொழில்முனைவின் மூலம் மனித மூலதனத்தால் தயாரிக்கப்படும் பனை ஓலைப் பொருட்களும் பயன்படுகின்றமை குறிப்பிடத்தக்கது. பனம்பொருள்சார் படைப்பாக்கத்தில் நபரின் கற்பனை, உழைப்பு, அழகுணர்வு, அதன் அழகியல் நுட்பங்கள் என்பவை பண்பாட்டுத் தளத்தில் அதற்கான அர்த்தப்பாட்டைப் பெறுமதி வாய்ந்ததாக மாற்றுகின்றது. இது அதனைப் படைக்கும் கலைஞர்களின் உழைப்பாகும்.

அவ்வாறே, சடங்கு நிகழ்வுகளில் பனம்பொருட்களின் தேவை மனிதத் திறனின் படைப்பாக்க வெளியில் பயன்பாட்டிலுள்ளது. மரணச் சடங்கு, திருமணச் சடங்கு, கோயில் சடங்குகள் போன்றவற்றில் இதன் பயன்பாட்டை நோக்கலாம். பண்பாட்டிற்காக உற்பத்தி செய்யும் பனை ஓலையின் பொருட்களில் பாய், பெட்டி என்பன முன்னிலை வகிக்கின்றன. கோயிற் சடங்குகளில் நெல், அரிசி, வாழைப்பழம், மடைக்குரிய பொருட்கள் போன்றவை எடுத்து சுத்தமாக வைப்பதற்கு ஓலைப் பெட்டி பயன்படுத்தப்பட்டு வருகின்றது.

ஒருவருக்கு பேய் போன்ற கெட்ட ஆவிகள் பிடித்துள்ளது என இனங் காணப்பட்டால் 'கழிப்புச் சடங்கு' செய்வது கிராமப்புறங்களில் வழக்கம். ஓலையினால் தயாரிக்கப்பட்ட பெரிய தட்டில் தயிர், கூழ், மதுபானம், மீன், இறைச்ச போன்றவற்றை வைத்து மடைவைப்பர். இவ்வாறான சடங்கிற்குத் தேவைக்காக தட்டு வகைகளைத் தன்னிடம் 
கேட்டுக்கொண்டதற்கு இணங்கத் தயாரித்துக் கொடுத்தாக வாதகல்மடுக் கிராமத்தில் சுய தொழிலுக்காக செய்யும் திருமதி எஸ்.பார்வதி கூறினார் (Parvathi, 18.11.2018).

பத்தசிக் கோயிலில் நெல்லுக்குத்தும் சடங்கு நடைபெறுவது குறிப்பிடத்தக்க சம்பிரதாயமாகும். அந்த வேளையில் அந்த நெல்லை 'நேர்த்தியாக எடுத்து பனை ஓலைப் பெட்டியிலே' வைப்பர். பின்னர் அதனைக் குத்தி எடுப்பதற்கும் ஓலைப் பெட்டியைப் பயன்படுத்துவது வழக்கமாக உள்ளது. அந்தப் பொருட்களை வாங்கும் பொழுது சடங்கிற்குரிய எண்ணப்பாட்டுடனே வாங்குகின்றமை குறிப்பிடத்தக்கது (Yogarani, 20.11.2018).

சடங்கின்போது நேர்த்திக் கடனை நிறைவு செய்வதற்காக 'அடையாளப்பெட்டி' எடுப்பர். இதனுள் நெல், பூ, பழம், பட்டு, பாக்கு, வெற்றிலை, கண்மணி, பலகாரம் முதலான பொருட்களை ஓலைப் பெட்டியினுள் வைத்து வெள்ளைத் துணியால் கட்டி கோயிலில் கொடுப்பது வழக்கம் (Pusani, 10.10.2019). இந்த உலகமயமாக்கல் சூழலில் ஓலைப் பெட்டியே இதற்குப் பயன்படுத்தப்படுவது சிறப்பம்சமாகும்.

மட்டக்களப்பில் திருமணச் சடங்கின்போது 'பெட்டி கொண்டு போதல்' எனும் நிகழ்வு இடம்பெறுவது வழக்கம். இந்தச் சந்தர்ப்பத்தில் ஓலைப் பெட்டியையே அந்தப் பெட்டி கொண்டு போதலுக்குப் பயன்படுத்துவர். அதனுள் உணவுப் பண்டங்களை வைத்துத் துணியால் கட்டி தலையில் வைத்துச் சுமந்து கொண்டு போகும் வழக்கம் படுவான்கரையில் இன்றும் உள்ளது (Rasakumari, 09.10.2018). அத்தோடு, இறந்தவர்களுக்குரிய 'உத்தியாக்களின் ${ }^{2}$ போது வைக்கப்படும் மடையில் சோறு, கறி ஏனைய உணவுப் பொருட்கள் ஆகியவற்றைப் பனை ஓலைப் பெட்டியிலே படைப்பர். இன்று மட்டக்களப்பில் கிராமப் புறங்களில் இந்தப் போக்கு நிலவுகின்றது. மரணச் சடங்கின்போது உடலை வைக்கும் கட்டிலில் ஓலைப் பாயை விரித்து வைப்பதும் நடைமுறையில் உள்ளது. வண்ணார், அம்பட்டர் சமூகத்தினருக்கு ஒவ்வொரு பாய் கொடுப்பதும் பழக்கம். தமிழர் மரபின் பண்பாட்டு வெளியில் பனை ஓலையின் வகிபங்கு அவர்களின் வாழ்வியலோடு ஓன்றரக் கலந்தது. பனை ஓலை மக்களின் வாழ்வின் குறியீட்டு அம்சமாக விழங்குகின்றது. பனை ஓலையால் பின்னப்படும் தோறணம் மங்கல, அமங்கல நிகழ்வுகளின் பொழுது இரண்டுக்கும் இடையிலான வேறுபாட்டை பிரதிநிதித்துவப்படுத்தும் வகையில் அலங்கரிக்கப்படும்.

திருமண நிகழ்வில் 'பந்திப்பாய்' விரித்தே அமர விடுவர். கோயில்களில் அன்னதானம் கொடுக்கும்போதும் இந்த பந்திப்பாயே பயன்படுத்தப்படுகின்றது. இதன் நீளமான அளவுத் திட்டம், இதற்குப் பயன்படுத்தப்படும் நிற அலங்காரம் ஆகியவற்றின் அழகுக்கேற்ப கொள்வனவின்போது இதன் கேள்வி அதிகரிக்கும். அவ்வாறே திருமணத் தம்பதிகளுக்கு 'கலத்தில் போடும்போதும்' ஓலைப் பாயிலே இருத்தி முன்னெடுக்கும் வழக்கமும் உண்டு (Saraswathi, 11.10.2018). தற்காலத்தில் திருமண நிகழ்விற்காக கைவினைப் பொருட்களான பனை ஓலையால் தயாரிக்கப்பட்ட மாலைகள், பூச்செண்டுகள் உத்தரவின் பெயரில் செய்து கொடுக்கப்படுகின்றது. இந்தச் சந்தர்ப்பத்தில் உற்பத்தி செய்யப்படும்போது பண்பாட்டு

\footnotetext{
2 உத்தியாக்கள் என்பது இறந்தவர்கள். உறவினர்கள் இறந்த தமது உறவினர்களுக்கு ஒ ஒவ்வொரு வருடமும் உணவுப் பொருட்களைப் படைத்து வணங்குவதும் அவர்கள் வந்து உண்டுவிட்டுப் போவதுமான நம்பிக்கையுண்டு. இவ்வாறு இறந்தவர்களுக்குப் படைக்கும் நிகழ்வு உத்தியாக்களுக்குச் செய்தல் என்று மட்டக்களப்பில் அழைக்கப்படுகின்றது.
} 
உணர்வு, நிகழ்வுகளின் பொருத்தப்பாடு, மணமகன், மணமகளின் ஆடை அலங்காரத்திற்குப் பொருத்தமான வர்ணத் தெரிவுகள் ஆகியவற்றுடன் உணர்வு பூர்வமாக தயாரிப்பதும் அதே உணர்வுடன் வாங்குவதும் இடம்பெறுகின்றது. இப்பொருட்கள் அழகுடன் அந்த இதழ்களை வெட்டி, நிறப் பயன்பாட்டுடன் சாயம் கலந்த ஓலைகளைப் பொருத்தமாகச் சேர்த்து கற்பனைத் திறன் வெளிப்பாட்டிற்கேற்ப தயாரிக்கப்படும்.

விரத காலங்களில் பூசைக்குரிய பொருட்கள் கொண்டு செல்வதற்கு மூடியுடன் கூடிய பெட்டிகள் தயாரிக்கப்படுகின்றன. இதனை 'மடைப்பெட்டி' கொண்டு செல்தல் என அழைப்பர் (Rasakumari, 09.10.2018). நிலமானிய சமூக அமைப்பில் பனை ஓலையுடனான கைவினைப் பொருட்கள் அதிகம் பயன்படுத்தப்படுகின்றமை நிலவுகின்றது. முதியோர்கள் கிராமப்புறங்களில் கச்சான், குரக்கன், இறுங்கு, நெல் போன்ற தானியங்களை அறுவடை செய்வதற்காக பயிரிட்ட பின்னர் கைப்பெட்டி, பெட்டி, பாய் போன்றவற்றைத் தாமே தயாரிக்கும் வழக்கம் உண்டு. நெல் அறுவடை முடிந்ததும் அந்த நெல்லை தூசி இன்றி தூற்றுவதற்கு ஓலைக் கைப் பெட்டி' பயன்படுத்தப்படுகின்றது. தைப் பொங்கல் காலங்களில் 'புதிர்' எடுப்பது ஓலைப் பெட்டியிலேயாகும். இது வேளாண்மை முற்றிப்பழுத்த காலங்களில் புது நெல்லை வீட்டிற்குக் கொண்டு வருவதற்கு முன்னர் விவசாயிகளால் மேற்கொள்ளப்படும். அப்போது நெற் கதிர்களைக் கொத்தாகக் கட்டி சுவாமி அறையினுள் வைப்பதற்கு இப்பெட்டியே பயன்படுத்தப்படுகின்றது.

பண்பாட்டுத் தளத்தில் சடங்கு சம்பிரதாயங்களின்போது பனை ஓலையினால் தயாரிக்கப்பட்ட கைவினைப் பொருட்கள் இன்றை உலகமயமாக்கல் ெருக்கடிக் காலத்திலும் கிராமப்புறங்களில் பயன்படுத்தப்படுகின்றமையை அவர்களது கூற்றுக்களில் இருந்து அறியமுடிகின்றது. இரப்பர் உற்பத்திப் பொருட்கள் மலிவாகக் கைகளுக்குக் கிடைத்தாலும் மிகத் தெளிவாக மனமுவந்து பனை ஓலைப் பொருட்களைப் பயன்படுத்த வேண்டிய இடத்தில் பயன்படுத்தப்படுகின்றது. மனித மூலதனப் பயன்பாட்டை அதிகரித்து பேண்தகு பொருளாதாரத்தை வலுப்படுத்த சமூகபண்பாட்டில் உள்ள சடங்கு சம்பிரதாயங்கள் முதன்மை வகிக்கின்றதை உணரலாம். சடங்கின் தேவை மனித சமுதாய ஆளுமையினால் உருவான பனம்பொருட்களின் மூலம் பூர்த்தி செய்யப்படுவதானது கலைத் தொழில்முனைவுக்கும் சடங்குசார் வாழ்வியலுக்குமான நெருங்கிய தொடர்பைக் காட்டுகின்றது.

பொருளாதார மதிப்பை உற்பத்தி செய்வதற்கு மனிதத் திறன் படைப்பாக்கம் பனை ஓலைப் பொருட்களில் வெளிப்படுவதனால், பண்பாட்டுப் பாரம்பரியத்தில் ம்ன்னிலை வகிக்கின்றது. தொழில்நுட்பத்தின் உச்ச தாக்கத்தின் அறிதலும், தெளிவும் அவசியம். காலனிய சிந்தனைக்குட்பட்ட புத்திஜீவிகளின் நவீன கல்விப் பார்வையினால் வலுப்பெற்ற முதலாளிகளால் உள்ளூர்ப் பொருளாதார முறைமை கட்டமைக்கப்பட்டுள்ளது. அவை மக்கள் சார்ந்த பன்முகப் பார்வையுடன் உருவாக்கப்பட வேண்டும். மனிதத் திறனின் வெளிப்பாடு சரியாகக் கையாளப்படும்போது பேண்தகு பொருளாதார வளர்ச்சி அதிகரிக்கும். இந்த வளர்ச்சி மனித மூலதனத்திற்குச் சிறப்பான எதிர் காலத்தினை கைத்தொழில் உறவுகளினூடாக அமைத்துக் கொடுக்க முடியும். 
சமகாலத்தில் 'பனம்பொருள் உற்பத்தி நிலையத்தில்' பயிற்சி பெற்றவர்கள் கலைத் தொழில்முனைவில் உற்பத்தி செய்கின்றபோது சிறிய முதலாளிகளால் கொள்வனவுக் கட்டளை செய்யப்படுகின்றது. இந்த நோக்கத்திற்காக தொழிலைச் செய்பவர்கள் வருமானத்தை மட்டும் முதன்மைப்படுத்துவதால் பண்பாட்டு மனவெள்பாடு குறைவடைவது இயல்பாகும். ஆனால், இதனை வாங்கும் தமிழர் தம் பண்பாட்டு உணர்வுடனே வாங்கிப் பயன்படுத்துவதையும் அதிகரிக்க வேண்டும்.

\section{பனம்பொருள் கலைத் தொழில்முனைவால் கிடைக்கும் வருமானம்}

மனித ஆளுமை கலைத் தொழில்முனைவில் பிரயோகிக்கப்படுவதனால் பேண்தகு அபிவிருத்திக்கான அடிப்படை வலுப்பெறுகின்றது. உழைப்பைச் சுரண்டுபவர்களின் கைகளில் அகப்படாதவாறு கிடைக்கும் பணம் உள்ளூரில் கையளிக்கப்பட்டு அம்மக்களல் சேமிக்கப்படுகின்றமை பொருளாதார வளர்ச்சியில் கைவினைக் கலை உற்பத்திப் பொருட்கள் பலவழிகளில் பெரும்பங்கு வகிக்கின்றது என்பதை உணர்த்துகின்றது. சிறுகைத்தொழில் என்ற ரீதியிலும் இதற்கென தனியான அமைச்சு கொண்டுவரப்பட்டு இதனை முன்னெடுக்கின்றமை குறிப்பிடத்தக்கதாகும். பனை ஓலையினால் தயாரிக்கப்படும் கைவினைப் பொருட்கள் தனிநபர்களுக்குப் பெரும் வருமானத்தைப் பெற்றுத்தருகின்றது. பண்பாட்டுத் தளத்திலும், அலங்கார வெளியிலும் இத்தொழிலுக்கு தனியிடம் உண்டு என்பதனால் மிகவும் சிறப்புற வளம் பெறவழிவகுக்கின்றது. இதன் உற்பத்தியாளர்களான கைவினைக் கலைஞர்கள் தமது பிழைக்கும் தொழிலாகவும், பகுதி நேரத் தொழிலாகவும் செய்து வருமானத்தைப் பெறுகின்றனர்.

பனம் பொருளுக்கு முதலீடு செய்யும்போது அது பண்பாட்டு முதலீடாகவும் விளங்குகின்றது. பண்பாடுசார்ந்த நிகழ்வுகள் பருவகாலத்தில் தமிழர் மத்தியில் வருவதனால் அதற்காகப் பனை ஓலையில் பாய், பெட்டி, கூடை, கைப்பெட்டி போன்றவற்றை விற்பதற்காகப் பயிற்சி நிலையத்தில் பயிற்சி எடுக்காத உள்ளூர் மூத்த கைவினைக் கலைஞர்களும் பயிற்சி எடுப்பவர்களும் வருகின்றனர். எந்தவித கட்டுப்பாடும் இன்றி சுதந்திரமாகவும், சுயமாகவும் தமது முதலீட்டை மேற்கொண்டு வருமானத்தைப் பெறுகின்றமை பேண்தகு வளர்ச்சியின் அடிப்படையாக அமைகின்றமை குறிப்பிடத்தக்கது. அதிக பெண்கள் இவற்றில் ஈடுபடுவதனால் தமது பெற்றோரையும், குடும்பத்தாரையும் பராமரிக்கும் வருமானம் வந்து கட்டுகின்றது. கோயில்த்திருவிழாக் காலங்களில் தாம் தயாரிக்கும் பொருட்களைத் தாங்களே விற்று அல்லது உறவினர்கள் மூலம் விற்றுப் பணம் பெறுகின்றனர். இவை முதலாளிகளின் கையில் பணம் செல்லாது தமது பணம் தமது கிராமத்தினுள் சுழல்வதை ஏற்படுத்துகின்றது. சடங்கினை நடத்துபவர்களும், ஏனைய வாழ்வியல் சம்பிரதாயங்களை மேற்கொள்பவர்களும் தமது சமூக பொருளாதார மேம்பாட்டிற்காகவே செலவு செய்கின்றனர். உற்பத்தியாளர்களும் அதற்காகவே முதலீடு செய்கின்றனர். எந்தவித சுரண்டலோ. கட்டுப்பாடோ இங்கு குறைவு எனலாம். 
மிகவும் திட்டமிட்டு கிராமப்புறங்களில் வயது முதிந்தவர்கள் இதனை சிறப்புற வடிவமைத்து விற்கின்றமை உள்ளூர் உற்பத்தியின் வருமானத்தை மேலும் பெருக்குகின்றது. இவை ஊக்குவிக்கப்பட வேண்டும்.

பனம்பொருள்சார் கலைத் தொழில்முனைவின் மூலமான கைவினைப் பொருட்களைத் தயாரித்து விற்பவர்களில் மற்றுமொரு சாரார் மட்டக்களப்பு மாவட்ட பனம்பொருள் அதிகார சபையின் கீழ் பயிற்சி பெற்ற உற்பத்தியாளர்களாவர். இவர்கள் வரன்முறையாகப் பயிற்சி பெற்று, செய்து கற்று பின்னர் உற்பத்தி நிலையங்களுக்கு மாற்றப்பட்டு உற்பத்தி நிலையங்களிலோ அல்லது வீட்டில் இருந்தோ பொருட்களை தயாரித்துக் கொடுப்பர். இவர்கள் மூலமும் சமூக பொருளாதாரம் கிடைக்கின்றது. பனம்பொருள் அதிகார சபையூடாகவே பொருள்தேவை என்று உத்தரவு பிறப்பிக்கும்போது அ அதைக் கொண்டு உற்பத்தியாளர்கள் தாம் உற்பத்தி செய்த பொருட்களைக் கொடுத்து பணம் பெறுவது வழக்கமாக உள்ளது.

இது பற்றி அவர்கள் கூறுகையில் மட்டக்களப்பு பனை அபிவிருத்திச் சபை 30 வீதக் கழிவிலேயே உற்பத்தியாளர்களிடமிருந்து கொள்வனவு செய்கின்றனர்' (Ranji, 12.05.2018) என்றும், 'அதனைச் செ்பவர்களுக்கு வருமானம் கிடைக்கின்றது' (Parimalam, 12.02.2019) என்றும் 'இங்கு விலையை உற்பத்தியாளர்கள் தீர்மானிப்பதில்லை. தயாரிக்கும் போது கஷ்டங்கள் ஏற்பட்டாலும் அதற்குரிய ஊதியம் கிடைப்பதில்லை' (Kavitha, 12.05.2018) என்றும் 'தாம் தயாரிக்கும் பொருட்களுக்கு காலம் தாழ்த்தியாவது பணம் கிடைக்கின்றது' என்றும் (Niro, 13.05.2018) கூறுகின்றனர். மனித மூலதனத்தைத் தமது படைப்பில் பிரயோகிப்பவர்கள் பதிவு செய்யாததால் ஓய்வூதியத்திட்டம், நஷ்ட ஈடு என்பவற்றைப் பெறுவதில்லை என்பதும் அறிதலுக்குரியது.

பனம்பொருள் அதிகார சபையினூடாக பத்து இலட்சத்திற்கு மேல் கொள்வனவுக் கட்டளை பிறப்பிக்கப்படுகின்றதாகவும் அதனை உற்பத்தி செய்யும் உற்பத்தியாளர்கள் மெதுவாகவே செய்கின்றனர் எனவும் மட்டக்களப்பு மாவட்ட பனை அபிவிருத்திச் சபை முகாமையாளர் கூறினார். இதனை அதிகரிக்கும்போது வருடாந்தம் பத்து இலட்சம் ரூபா பெறுமதி வரையான பொருட்களை உற்பத்தியாளர்களிடம் இருந்து கொள்வனவு செய்யக் கூடியதாக இருக்கும் எனத் தெரிவித்தார். அத்தோடு, 'மட்டக்களப்பு மாவட்டத்தில் 2016ஆம் ஆண்டு வெளிநாட்டுச் சந்தை வாய்ப்புக் கிடைத்ததனால் 6 மில்லியன் ரூபாவும், 2017இல் உள்நாட்டு சந்தையில் 3.5 மில்லியன் ரூபாவும், 2018இல் 4 மில்லியன் ரூபாவும் வருமானம் கிடைத்துள்ளதாகக் கூறினார் (Vijayan, 05.02.2019).

பயிற்சி எடுத்தவர்கள் அதிகமாகப் பெண்களேயாவர். இவர்கள் தனியாகவும் தமது வருமானத்தைப் பெற்றுக் கொள்கின்றனர். பெண்கள் தமது உறவினர்களுடன் சேர்ந்து நேரடியாகத் திருமண 'கேக் கூடையைத்' தயாரித்துக் கொடுத்தும் வருமானத்தைப் பெறுகின்றமை குறிப்பிடத்தக்கது.

மட்டக்களப்பில் பனம்பொருள் உற்பத்தியை ஊக்குவித்து அதன்மூலம் வருமானத்தைப் பெறவாய்ப்புண்டு. அதிகமானோர் சுயமாகத் தமது பொருளாதாரத்தைத் தொழில்முனைவுடன் 
ஈட்டிக் கொண்டும் இருக்கின்றனர். பண்பாட்டு ரீதியாக உற்பத்தி செய்பவர்கள் மத்தியிலும் தமது வாழ்வாதாரத்திற்குரிய வருமானம் கிடைக்கின்றது.

இதனை நோக்கும்போது மட்டக்களப்பு மாவட்டத்தில் பனம்பொருள்சார் கலைத் தொழில்முனைவில் மனிதத் திறனும் ஆளுமையும் பயன்படுத்தப்படுவதனால் தயாரிக்கப்படும் கைவினைப் பொருட்கள் பண்பாடு, உற்பத்தி, அலங்காரம், னைவினைக்கலை போன்ற தளங்களில் மிகவும் வலுவாக உள்ளூர் வருமானம் சார்ந்து முன்னெடுக்கப்படுகின்றது. எனினும், உலகமயமாக்கலின் தாக்கம் பெரும் பாதிப்பை ஏற்படுத்துகின்றது. பனை அபிவிருத்தி அதிகாரசபை படுவான்கரையில் விஸ்தரிப்பது முக்கியமான கடமைப்பாடாகும். அத்தோடு, உற்பத்தியைத் தூண்டும் அதிகார சபையும், உற்பத்தி செய்யும் உற்பத்தியாளர்களும் பல சவால்களையும் எதிர்கொண்டு வருகின்றமையும் குறிப்பிடத்தக்கது.

\section{மட்டக்களப்பில் பனம்பொருள் உற்பத்தியாளர்கள் எதிர்கொள்ளும்} சவால்கள்

பனம்பொருள்சார் மனித மூலதன பிரயோகத்தின் வெளியில் உற்பத்தி தொடர்பான பல சவால்களும் பிரச்சினைகளும் முன்மொழியப்படுகின்றன. அவை உற்பத்தியாளர்கள், உற்பத்தியை ஊக்குவிப்பவர்கள் என அமைகின்றது.

இன்றைய காலங்களில் உலகமயமாக்கலின் உச்சத்தினால் பல்தேசியக் கம்பனிகள் அந்தந்தப் பிரதேசங்களில் புதிய பல பொருட்களை உற்பத்தி செய்தோ அல்லது இறக்குமதி செய்தோ அறிமுகப்படுத்துகின்றது. அவற்றுள் இரப்பர் பொருட்களும் முக்கியமானவை. இவை மக்கள் மத்தியில் பனை ஓலைக் கைவினைக்கலை உற்பத்தியிலும் அதன் கேள்வியிலும் தாக்கத்தை ஏற்படுத்தியுள்ளது. இதனால், பெரிதும் உற்பத்தி பின்னடைகின்றது. தொழில்முனைவுக்கான உற்பத்தி ஊக்குவிப்பாளர்கள், உற்பத்தியாளர்கள் ஆகியோர் எதிர்கொள்ளும் சவால்கள்:

- அலங்காரப் பொருட்கள் பற்றித் தெரியாதவர்கள் ஆர்வத்துடன் பயிற்சி எடுக்க வருகின்றனர். பயிற்சிக் காலங்களில் தம்மை முழுமையாக ஈடுபடுத்தாது இடைவிலகிக் கொள்கின்றனர். இதனால் இக்கற்கையைத் தொடர்ந்து பின்பற்றுவது கடினமாகும்.

- மூலப் பொருளை சில இடங்களில் பெற்றுக்கொள்வது கடினம். உத்தரவு கொடுத்ததால் தயாரிக்கின்றோம் எனக் கூறி பின்னர் பொருட்களைக் கேட்கும் பொழுது 'ஓலை வரல்ல, ஓலை வெட்டல்ல' எனப் பின்வாங்குகின்றமை.

- மூலப் பொருள் பற்றிய விளக்கமின்மை. சல இ இடஙகளில் இலகுவாகக் கிடைப்பதனால் இதற்குப் பெறுமதி இல்லை என நினைக்கின்றமை.

- கைவினை உற்பத்தியாளர்கள் தயாரிக்கும் பொருள் தரமில்லை, அதன் முடிவு சரியில்லை, உற்பத்தி நிலையத்தில் கொள்வனவு செய்பவர்கள் திருப்பி அனுப்புகின்றமையால் மனித மூலதனம் வீணாகின்றது. இது நவீன அறிவின் காலனிய சிந்தனை வெளிப்பாடாகும். 
- ஓலைகளைத் தேவைக்கேற்ப வார்ந்தெடுப்பதற்குரிய இயந்திரம் வாங்குவதற்கு உற்பத்தியாளர்களிடம் வசதியின்மை காணப்படுகின்றமை. இதனால் விரைவாகச் செய்து கொடுக்க முடியாதுள்ளமை.

- மழைக் காலங்களில் பனை ஓலையில் பொருட்கள் செய்வதும், சேர்ப்பதும் சவாலாகும். பனை ஓலையை வெட்டி, காயவிட்டு பொருத்தமான அளவுடன் சீவி எடுப்பது மிகவும் கடினமாகும். ஏனெனில், ஓலைகள் காயாது விட்டால் பூஞ்சனம் பிடித்து நிறமாற்றத்தைத் தரும்.

எனினும் சமூக, பண்பாட்டு வாழ்வியல் தளங்களில் மிகவும் பெறுமதி வாய்ந்ததாக கலைத் தொழிலமமுனைவின் மூலமான மனித மூலதனப் படைப்பாக்கப் பிரயோகம் பயன்படுத்தப் படுகின்றமையினால் நிலைபேறான வருமானத்தை ஈட்ட வழிவகுக்கின்றது.

\section{பரிந்துரைகள்}

அரசாங்கம் இந்தக் குடிசைக் கைத்தொழிலை ஊக்குவிப்பது குறைவாக உள்ளது. பயிற்சி எடுத்துவிட்டு வீட்டில் தொழிலின்றி இருக்கின்றவர்களுக்கு வசதிகளைச் செய்துகொடுத்து மேலும் வழிப்படுத்தி ஊக்குவிப்பது மிகவும் அவசியமான ஒன்றாகும். தனியார் நிறுவனங்கள் பயிற்சியை நடாத்தும்போது மாதாந்தம் பயிற்சி பெறுனர்களுக்கு ஒரு தொகை பணம் கொடுப்பனவாக வழங்கி பயிற்சியளித்துள்ளதன் கைவினைப் பொருட்களைத் தயாரிப்பதற்கான பயிற்சி பெறுநரும் அவ்வாறு எதிர்பார்க்கின்றனர். இதனால் இந்த நபர்களின் ஆளுமை விருத்திக்குப் பங்களிப்புச் செய்வது அவசியமாகும்.

விரும்பிய அளவு வருமானம் கிடைப்பது குறைவாக உள்ளதாகப் பனம்பொருள்சார் தொழில்முனைவாளர்கள் குறிப்பிடுகின்றனர். சமகால முதலாளித்துவத்தின் பரவலாக்கத்தினால் உருவான தொழில் மையங்களில் அதிகம் வருமானம் கிடைக்கும் என்பதனால் அதனை நாடுகின்றனர். இதனைக் கருத்தில் கொண்டு மனித மூலதனத்தைப் பொருத்தமாகப் பிரயோகிக்க அரச நிறுவனங்கள் உள்ளூர் நிலையங்களில் வலுவாகக் கவனம் செலுத்த வேண்டும்.

மட்டக்களப்பில் கடந்த ஒன்பது ஆண்டுகளாக 'பனை அபிவிருத்தி அதிகார சபை' இயங்கி வருகின்றமையை அறிய முடிகின்றது. எனினும், பயிற்சி நிலையங்களை விஸ்தரிப்பதற்கும், உற்பத்திப் பொருட்களை உற்பத்தி நிலையங்களில் இருந்து விற்பனை நிலையங்களுக்கு சேகரிப்பதற்கும் பேக்குவரத்து ஏற்பாடுகள் குறைவு.

சுயமாகக் கிராமத்தவர்கள் தமது தொழில்முனைவை முன்னெடுத்து அறிவையும் ஆற்றலையும் பயன்படுத்துவது காலத்தின் தேவை. இவை பண்பாடு, கலை, உற்பத்தி ஆகிய தளங்களில் இப்பொருட்களைப் பயன்படுத்த மனித மூலதனத்தைப் பிரயோகிக்க வேண்டும்.

\section{முடிவுரை}

கலைத் தொழில்முனைவின் முன்னெடுப்பில் மனித மூலதனம் பெறுமதி வாய்ந்தது. பனம்பொருள்சார் உற்பத்திகள் மனித திறன், படைப்பாக்கம், ஆளுமை, அழகியல் உணர்வு என்பன வலுவாகப் பயன்படுத்தப்படுவதனால்தான் பண்பாட்டிலும், சடங்கிலும், அலங்கார 
வெளிப்பாட்டிலும் அதன் பாவனை பின்னிப் பிணைந்துள்ளது. சமுதாய அழகியல் அதன் பண்பாட்டு வெளியில் பிரதானப்படுத்தப்படுவதனால் மனித ஆளுமை பிரயோகம் முதன்மை அடைந்து கலைத் தொழில்முனைவில் தாக்கம் பெற்று பேண்தகு பொருளாதாரத்தை வலுப்படுத்துகின்றது எனலாம். மட்டக்களப்பில் இதன் செயற்பாட்டு வெளிப்பாடு எவ்வாறு அமைகின்றன என்பதை இவ்வாய்வினூடாக அறியமுடிகின்றது.

\section{References}

Karl Marx, (1998), Capital, Edited by Krishnaiah, Translated by Thiyagu, 1st ed., New Century Book House, Chennai, India.

Vijayan, T., (05.05.2018), Interview, District Manager, Palm Development Board, Palameenmadu, Batticaloa, Sri Lanka.

Niro, S., (13.05.2018), Interview, Development Officer, Barrow, Sri Lanka.

Thangaraja, S., (14.06.2018), Interview, farmer, VC Road, Vantharumoolai, Sri Lanka.

Rasakumari, T., (09.10.2018), Interview, Business, Nagamunai, Ambalanthurai, Sri Lanka.

Saraswathi, S., (11.10.2018), Interview, Homework, Nagamunai, Ambalanthurai, Sri Lanka.

Parvathi, S., (18.11.2018), Interview, Homework, Vagal Madu, Sri Lanka.

Yogarani, T., (20.11.2018), Interview, Homework, VC Road, Vantharumoolai, Sri Lanka.

Kavitha, S., (12.05.2018), Interview, Palm Decoration Work, Palameenmadu, Sri Lanka.

Parimalam, P., (12.05.2018), Interview, Palm Decoration Work, Palameenmadu, Sri Lanka.

Ranji, P., (12.05.2018), Interview, Palm Decoration Work, Palameenmadu, Sri Lanka.

Pusani, S., (10.10.2019), Interview, Homework, Arumugam residence, Sri Lanka.

\section{Funding: NIL}

Acknowledgement: NIL

Conflict of Interest: NIL

About the License:

\section{(c) (1)}

Attribution 4.0 International (CC BY 4.0)
(C) The author 2021. The text of this article is licensed under a Creative Commons Attribution 4.0 International License 